\title{
A Comparative Study of Fatigue Energy Dissipation of Additive Manufactured and Cast AlSi10Mg Alloy
}

\author{
Chunxia Yang ${ }^{1}$, Ke Zhu ${ }^{1}\left(\right.$, , Yayan Liu ${ }^{1}$, Yusheng Cai ${ }^{2}$, Wencheng Liu ${ }^{2}$, Keming Zhang ${ }^{2}\left(\mathbb{D}\right.$ and Jia Huang ${ }^{2,3, *}$ \\ 1 COMAC Flight Test Center, Shanghai 201323, China; yangchunxia@comac.cc (C.Y.); zhuke@comac.cc (K.Z.); \\ liuyayan@comac.cc (Y.L.) \\ 2 School of Mechanical Engineering, University of Shanghai for Science and Technology, Shanghai 200093, \\ China; 202271443@st.usst.edu.cn (Y.C.); wencheng.liu@usst.edu.cn (W.L.); zhangkeming@usst.edu.cn (K.Z.) \\ 3 School of Civil Aviation, Northwestern Polytechnical University, Xi'an 710072, China \\ * Correspondence: jia.huang@usst.edu.cn
}

Citation: Yang, C.; Zhu, K.; Liu, Y.; Cai, Y.; Liu, W.; Zhang, K.; Huang, J. A Comparative Study of Fatigue Energy Dissipation of Additive Manufactured and Cast AlSi10Mg Alloy. Metals 2021, 11, 1274 https://doi.org/10.3390/ met11081274

Academic Editor: Filippo Berto

Received: 1 July 2021

Accepted: 9 August 2021

Published: 12 August 2021

Publisher's Note: MDPI stays neutral with regard to jurisdictional claims in published maps and institutional affiliations.

Copyright: () 2021 by the authors. Licensee MDPI, Basel, Switzerland. This article is an open access article distributed under the terms and conditions of the Creative Commons Attribution (CC BY) license (https:// creativecommons.org/licenses/by/ $4.0 /)$.

\begin{abstract}
In this paper, the fatigue energy dissipation of Gravity Casting (GC) and Laser-based Powder Bed Fusion (LPBF) AlSi10Mg alloys under cyclic loading are investigated. The increase in surface temperature related to the energy dissipation effect is decoupled and used to predict the fatigue limits of GC and LPBF AlSi10Mg alloys as being 55.8\% UTS and 33.9\% UTS, respectively. The energy dissipation rate is obtained by solving the one-dimensional thermal diffusion problem. This energy dissipation is separated into related and unrelated fatigue damage using polynomial function fitting. The energy dissipation related to fatigue damage for LPBF specimens is observed to be higher than that of GC specimens, which indicates worse fatigue performance. The fatigue damage entropy is employed to predict the fatigue life of both GC and LPBF AlSi10Mg alloys, which has a good agreement with the results of a traditional fatigue experiment.
\end{abstract}

Keywords: AlSi10Mg alloy; additive manufacture; energy dissipation; thermal analysis; fatigue property assessment

\section{Introduction}

With the ever-increasing demands of quick response, high performance, high precision, light weight, and integrated manufacturing in the aviation industry, metal-forming technology faces higher challenges. As a short-process and highly flexible manufacturing technology driven by 3D model, Additive Manufacturing (AM) can effectively settle the manufacturing difficulties of complex metal components and enhance the design freedom for new aircrafts [1]. One of the most popular AM technologies is Laser-based Powder Bed Fusion (LPBF), which is a processing method combining a high energy laser beam as the heat source and metal powder as the raw material [2]. It can quickly and directly form final metal components and provides an excellent solution for reducing structural weights and machining costs in the design of new aircrafts.

Currently, the most widely used metallic material for LPBF in aviation industry is titanium and aluminum alloys. Between these two alloys, aluminum alloy is particularly suitable for manufacturing large structural components as well as thin parts in civil aircraft because of its low density, high specific strength, high thermal and electrical conductivity, excellent oxidation, and corrosion resistance. AlSi10Mg, as the material with the largest production of cast aluminum alloy, has a low thermal expansion coefficient, good formability, and excellent welding and casting performance, which is frequently used in the field of laser additive manufacturing such as LPBF. Therefore, the mechanical properties of 3D printing forming AlSi10Mg components has attracted much research interests in recent years.

Previous research [3] shows that the average yield strength is $10 \%$ higher and that the ultimate tensile strength is about $20 \%$ higher for the LPBF-made AlSi10Mg than that of cast 
AlSi10Mg, while the fatigue property of LPBF-made AlSi10Mg is significantly worse than the castings [4]. The fatigue property of LPBF-formed parts is affected by different kinds of defects, such as pores, cracks, inclusions, non-melting, and spheroidization [5]. The formation of defects can be influenced by various processing factors, listed as laser power, scanning rate, scanning spacing, powder layer thickness, protective atmosphere, post-heat treatment, etc. As known, the fatigue property is an important consideration during the structural design of aircrafts, which is closely related to flying safety. The fatigue properties of LPBF-made parts with different processing factors could have significant differences. However, using a traditional fatigue testing method to evaluate the fatigue property is rather time consuming and costly, which limits the application of LPBF-made parts.

The essence of fatigue behavior can be considered an irreversible process of mechanical property degradation [6], which is accompanied by heat generation and dissipation. The data of this thermal energy contain rich information on the fatigue damage evolution, which can be used to evaluate the fatigue property efficiently. With the rapid development of infrared detector technology, the surface temperature variation can be recorded with high accuracy. This temperature variation is linked to a fatigue hysteresis cycle, which can be accurately assessed by Digital Image Correlation (DIC) [7]. A thermographic methodology was first used to rapidly determine the fatigue limit for steels [8-10]. Then, the relationship between fatigue damage and crack with heat energy dissipated energy was further analyzed [11-13]. Recently, energy dissipation-based methods have increasingly been used to predict the fatigue property based on self-heating data [9,10,14-22]. According to the second law of thermodynamics, the energy dissipation of a system leads to an increase in entropy. Recent research shows that the total accumulated entropy generation of the whole fatigue life is constant and independent of geometry, load, and frequency in terms of medium and high cycle fatigue $[23,24]$. Those findings enable researchers to use cumulated thermodynamic entropy to obtain an S-N curve in a short time for several kinds of materials [25-29]. Hence, it would be of great significance if this method can be applied on $\mathrm{AM}$ aluminium alloy for rapid fatigue quality assessment. Furthermore, the combination of microstructure design and rapid fatigue property prediction obviously accelerates the product development process [30].

However, fewer studies are carried out on the fatigue energy dissipation of AM aluminium alloy. As mentioned before, the internal defects in the micro-structure of AM aluminium alloy are more complex than that of castings. It is still unclear how those defects affect the relationship between fatigue damage evolution and energy dissipation. According to previous works [31-33], the heat energy can be generated by both internal friction (damage evolution unrelated) and irreversible changes (damage evolution related). It is also unknown whether the initial defects have an influence on the internal friction. Therefore, it is valuable to conduct a comparative study of AM and cast aluminium alloy to obtain a better understanding of the thermal-dynamic mechanism and to know whether the fatigue properties can be assessed by the same energy dissipation-based methodology.

In the present paper, static tests of LPBF and cast AlSi10Mg alloy are first carried out to obtain the ultimate tension stress. Then, fatigue tests of two kinds of AlSi10Mg alloys under various amplitudes of applied stress are conducted with an infrared camera recording the thermographic data and digital image correlation system, measuring strain changes. Thereafter, the energy dissipation rate per unit volume is figured out by solving the local thermodynamic equation of state. Following this, the energy dissipation related to internal friction and fatigue damage evolution is separately analyzed and compared for those two kinds of AlSi10Mg alloys. Lastly, the accumulated entropy related to damage evolution is calculated to see if it can be used to predict the fatigue property of LPBF and cast AlSi10Mg alloy in a short time. 


\section{Materials and Experiments}

\subsection{Raw Materials}

The raw material used for LPBF manufacturing was AlSi10Mg prealloyed powder, which was provided by FalconTech Co., Ltd., Wuxi, China. The powder has a nearly spherical morphology with an average particle size of $26.9 \mu \mathrm{m}$. The Gravity Cast (GC) AlSi10Mg was supplied by Baoji ZhiYi Titanium Manufacturing Co.,Ltd., Baoji, Shaanxi, China. Cylindrical GC AlSi10Mg samples with a radius of $15 \mathrm{~mm}$ and height of $120 \mathrm{~mm}$ were prepared by machining, as shown in Figure 1a. The chemical compositions of the LPBF and GC AlSi10Mg alloys are shown in Table 1.

Table 1. Mean chemical composition (wt\%) of AlSi10Mg produced by Laser-based Powder Bed Fusion (LPBF) and Gravity Casting (GC).

\begin{tabular}{ccccccccc}
\hline & $\mathbf{S i}$ & $\mathbf{M g}$ & $\mathbf{F e}$ & $\mathbf{M n}$ & $\mathbf{C u}$ & $\mathbf{Z n}$ & $\mathbf{P b}$ & $\mathbf{A l}$ \\
\hline LPBF & 9.544 & 0.338 & 0.137 & 0.002 & 0.001 & $<0.002$ & $<0.002$ & Balance \\
\hline GC & 9.375 & 0.313 & 0.422 & 0.227 & 0.066 & 0.053 & 0.009 & Balance \\
\hline
\end{tabular}

\subsection{LPBF Process}

The cylindrical LPBF AlSi10Mg samples with the same dimensions as the GC samples were fabricated by a 3D printing machine called Hanbang 280, which was equipped with a $500 \mathrm{~W} \mathrm{Yb-fibre} \mathrm{laser.} \mathrm{It} \mathrm{should} \mathrm{be} \mathrm{noted} \mathrm{that} \mathrm{LPBF} \mathrm{AlSi10Mg} \mathrm{can} \mathrm{be} \mathrm{very} \mathrm{anisotropic}$ and that the mechanical property of LPBF AlSi10Mg can be also influenced by printing hatching, later thickness, and power density. Thus, in this paper, the printing direction and all of the printing parameters remain unchanged for all LPBF AlSi10Mg specimens. After several previous trials for process parameter optimization, the LPBF process in this work was conducted using a laser power of $300 \mathrm{~W}$ and a scan speed of $1200 \mathrm{~mm} / \mathrm{s}$ under the protective atmosphere of Ar. The layer thickness was $30 \mu \mathrm{m}$, and the hatch spacing was set as $140 \mu \mathrm{m}$. The substrate was pre-heated to $100^{\circ} \mathrm{C}$ to reduce the internal residual stress and deformation caused by rapid cooling process. The LPBF cylindrical samples were built along the $\mathrm{YZ}$ direction, as shown in Figure $1 \mathrm{~b}$.
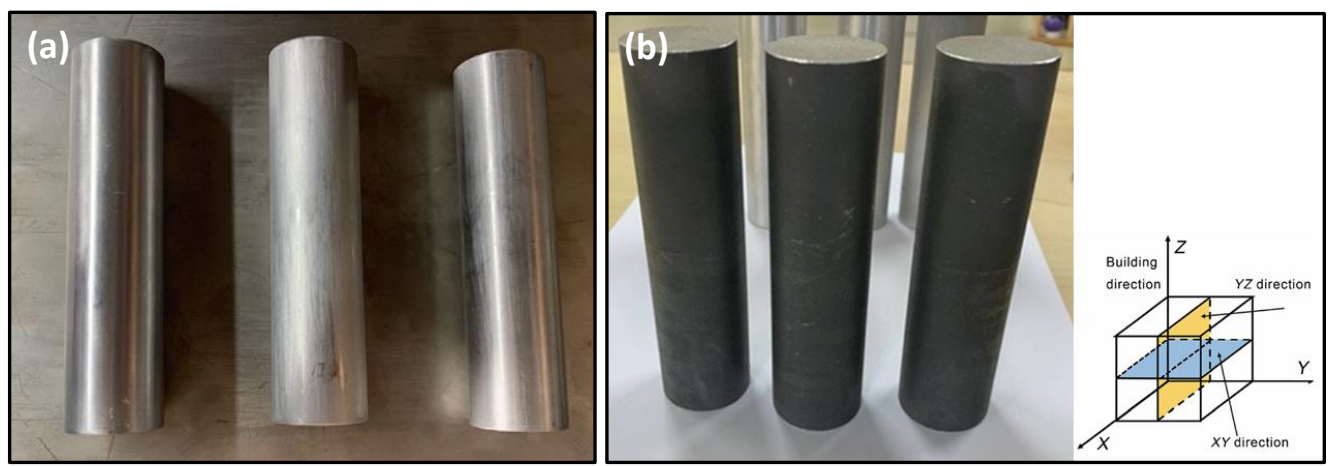

Figure 1. Pictures of the cylindrical AlSi10Mg samples: (a) GC (Gravity Casting) samples; (b) LPBF (Laser-based Powder Bed Fusion) samples.

\subsection{Specimen Preparation}

The static and fatigue specimens were machined per standards ASTM E8M-04 and ASTM E466-15, respectively. The specific geometries of are shown in Figure 2a,b. Both the GC and LPBF fatigue specimens were polished to ensure that the roughness of fatigue specimen was less than $0.8 \mu \mathrm{m}$. In order to measurement requirements of IR camera and DIC system, the specimens were painted with matte black on one side and with black speckle on white primer on the other side using spray paint. According to the property of paint, the emissivity was given as 0.98 . The photos of prepared static and fatigue specimens of GC and LPBF are shown in Figure 2c,d. 


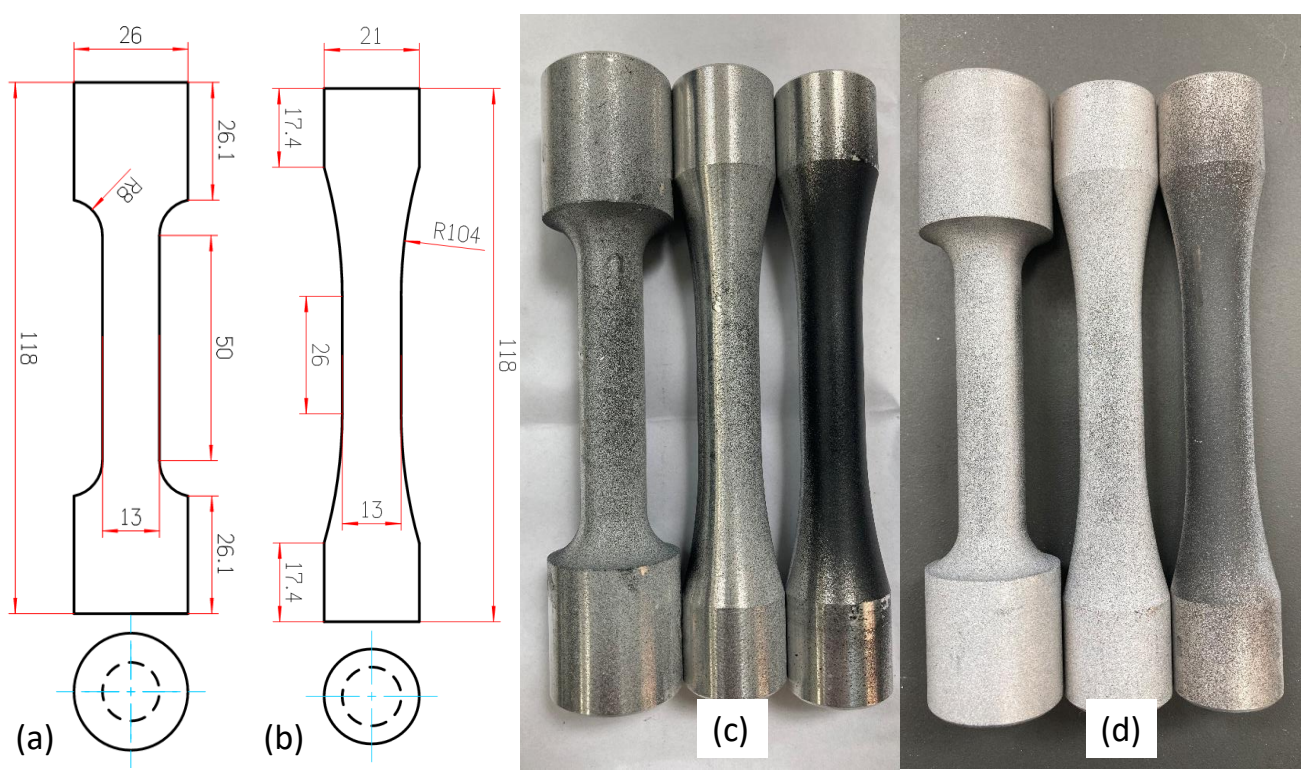

Figure 2. Geometries and pictures of static and fatigue specimens of GC and LPBF: (a) geometry of the static specimen, (b) geometry of the fatigue specimen, (c) static and fatigue specimens of GC, and (d) static and fatigue specimens of LPBF.

\subsection{Experimental Setup}

Quasi-static tensile testing was conducted until final breakage of the specimen in order to determine the Ultimate Tensile Stress (UTS). The experiments were carried out using a universal testing machine INSTRON 5985, manufactured by INSTRON Co., Ltd., Grove City, PA, USA, $250 \mathrm{kN}$ load capacity) with the Digital Image Correlation (DIC) technique. The hardware components of the DIC system consisted of three parts: (1) two CMOS cameras $(1080 \times 1920$ pixels, 8 bits $)$ for image acquisition, (2) a tripod for support, and (3) a computer for software installation. The camera was located about $710 \mathrm{~mm}$ from the specimens' surface, and the spatial resolution was about $48 \times 48 \mu \mathrm{m}^{2}$. The adopted sampling frequency for acquiring DIC data during the tensile tests was set as $5 \mathrm{~Hz}$. The quasi-static tensile tests were carried out piloted on the displacement, and the experimental crosshead velocity was set as $1.0 \mathrm{~mm} / \mathrm{min}$. The tensile force was recorded by the sensor of INSTRON 5985, and the strain field was calculated from the captured images series by using post-processing software called DICe. Then, the modulus of elasticity can be determined based on the data measured above.

Uniaxial fatigue tests of various applied stress amplitudes were carried out to obtain the self-heating data of GC and LPBF specimens. All fatigue tests were conducted under load-control mode at room temperature by using a servo-hydraulic fatigue testing system (INSTRON 8801-2, manufactured by INSTRON Co., Ltd., Grove City, PA, USA, 100 kN capacity of load). For each load level, the specimens were subjected to a constant loading amplitude sinusoidal wave-form with a frequency of $10 \mathrm{~Hz}$ and a stress ratio of -1 . An infrared camera (IRay A8Z3) with VOx sensors, $384 \times 288$ pixels, and $60 \mathrm{mK}$ thermal sensitivity was employed to record the variation of temperature on the surface of specimens in situ and in real-time. The infrared system was composed of the camera connected to a laptop, and the software called InfiView was used for control, configuration, and data post-processing. The sampling frequency of infrared camera was set as $25 \mathrm{~Hz}$. The detailed experimental setup is shown in Figure 3. A reference specimen was placed near the testing specimen in order to monitor the temperature change in ambient temperature. The IR camera was located in front of the specimens at a distance of $310 \mathrm{~mm}$ to record the temperature of both the reference and trial specimen. For each cyclic loading stress, the specimen was only tested for 3000 loading cycles, which was sufficient for recording the 
stabilized rise in temperature. In order to show this clearly, a scheme of the experimental and data processing procedures is given in Figure 4.

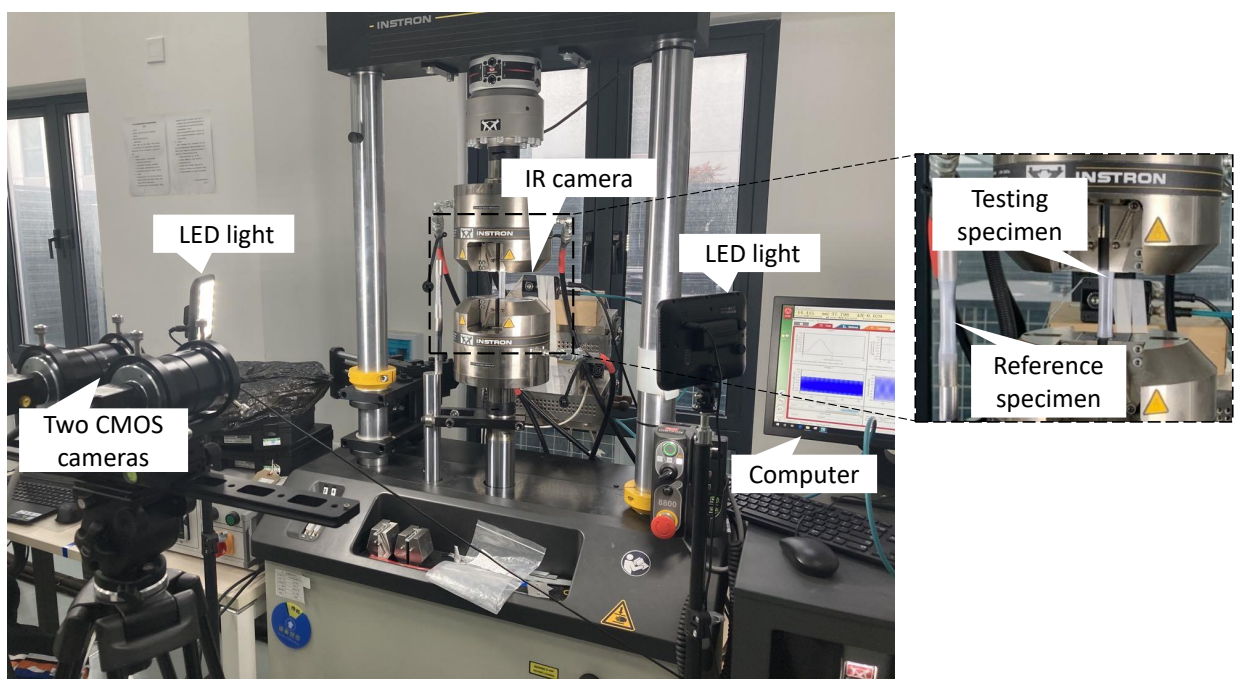

Figure 3. Experimental setup of the fatigue test with an infrared camera and a Digital Image Correlation (DIC) system of two Complementary Metal Oxide Semiconductor (CMOS) cameras.

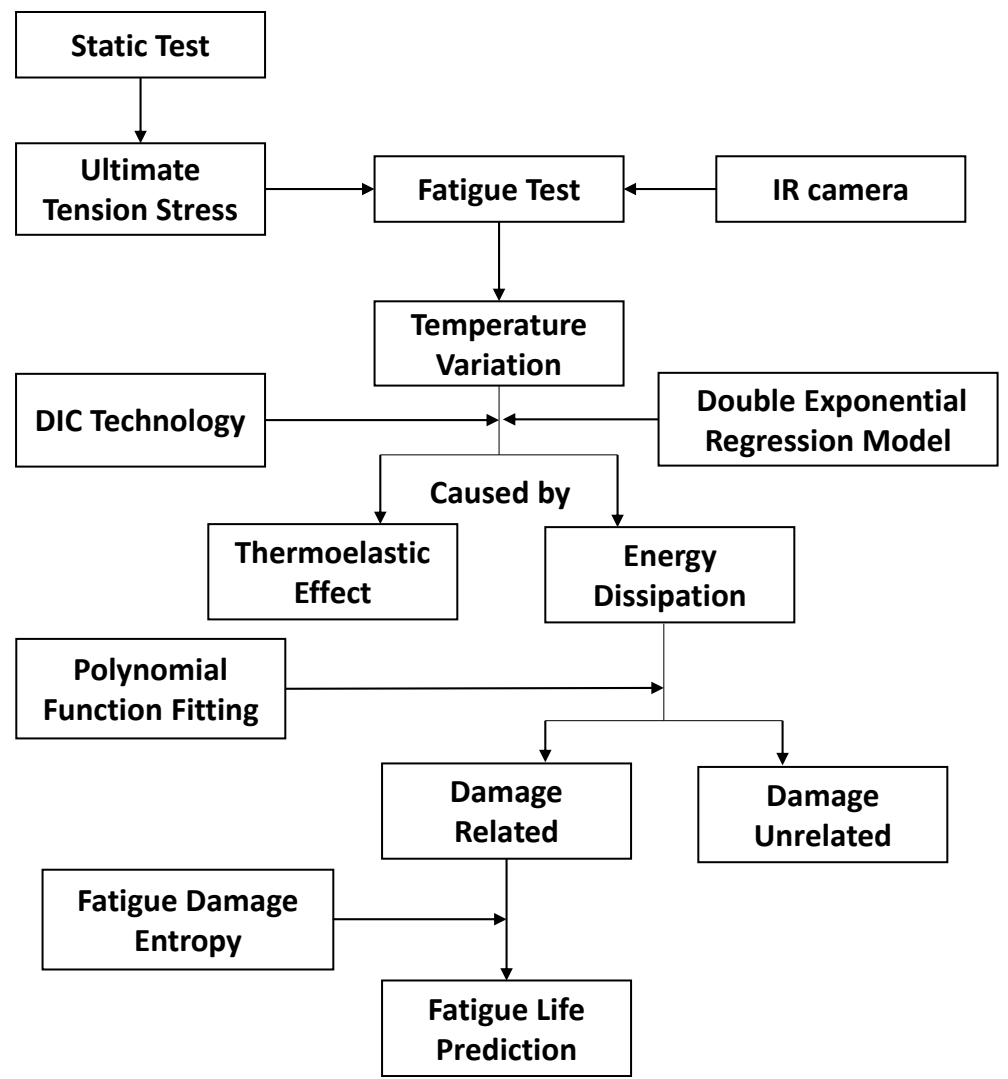

Figure 4. Scheme of the experimental and data-processing procedures.

\section{Fatigue Energy Dissipation Calculation}

As a macroscopic representation of energy dissipation, the surface temperature not only is determined by the evolution of fatigue damage but also is influenced by the external environment. Thus, it is essential to use temperature data to reconstruct different kinds 
of energy sources under fatigue load. Combining both the first and second principles of thermodynamics, the local thermodynamic equation of state can be expressed as follows:

$$
\rho C \dot{T}+\operatorname{div} J_{q}=d_{1}+s_{\text {the }}+s_{\text {ic }}+r_{\mathrm{e}}
$$

where $\rho$ represents the mass density, $C$ is the specific heat, $T$ is the local temperature, $J_{q}$ is the heat flux through the boundary, $d_{1}$ is the intrinsic energy dissipation, $s_{\text {the }}$ is the thermoelastic source, $s_{\mathrm{ic}}$ is the "internal" coupling source, and $r_{\mathrm{e}}$ is the external heat supply. $d_{1}, s_{\text {the }}$, and $s_{\text {ic }}$ are further defined as follows:

$$
\begin{gathered}
d_{1}=\left(\sigma-\rho \frac{\partial \Psi}{\partial \epsilon}\right): \dot{\epsilon}-\rho \frac{\partial \Psi}{\partial \alpha} \cdot \dot{\alpha} \\
s_{\text {the }}=\rho T \frac{\partial^{2} \Psi}{\partial T \partial \varepsilon}: \dot{\varepsilon} \\
s_{\text {ic }}=\rho T \frac{\partial^{2} \Psi}{\partial T \partial \alpha} \cdot \dot{\alpha}
\end{gathered}
$$

where $\sigma$ is the Cauchy stress tensor, $\varepsilon$ is the small perturbation hypothesis strain tensor, $\Psi$ is the Helmholtz free energy, and $\alpha$ is the components of a vector of $n$ internal variables $n$ $\alpha_{i}(i=1, \ldots, n)$.

For homogeneous uniaxial fatigue tests in this study, the temperature variation is mainly (almost 90\%) induced by the thermoelastic coupling effect and by the energy dissipation effect. Thus, Equation (1) can be approximately simplified as follows [31,34,35]:

$$
\rho C\left(\frac{d \theta}{d t}+\frac{\theta}{\tau_{\text {eq }}}\right) \approx s_{\text {the }}+d_{1}
$$

where $\theta$ is the temperature variation at the center of the specimen gauge part and where $\tau_{\text {eq }}$ characterizes all local heat losses. We can define $\theta_{\text {the }}$ and $\theta_{d}$ induced by $s_{\text {the }}$ and $d_{1}$, respectively. During the fatigue test, a large number of studies $[17,33,36]$ showed that there are three phases in the thermodynamic behavior, which are shown in Figure 5. The temperature variation of the specimen subjected to homogeneous fatigue loading is observed to have three phases. In Phase I, the temperature increased with loading cycle number and then reached a nearly equilibrium temperature. During Phase II, the average temperature remains almost unchanged and the thermoelastic coupling effect and energy dissipation effect stay stable. Phase II also counts for most of the fatigue life. Phase III rapidly increases until fatigue failure in a few loading cycles, and the evolution of $\mathrm{T}$ in this phase usually lacks regularity. Therefore, Phase II is chosen to study the thermodynamic behavior of the LPBF and GC AlSi10Mg alloys.

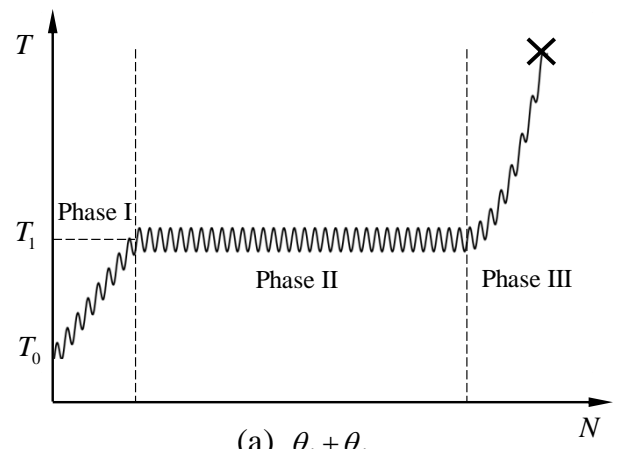

(a) $\theta_{\mathrm{d}}+\theta_{\text {the }}$

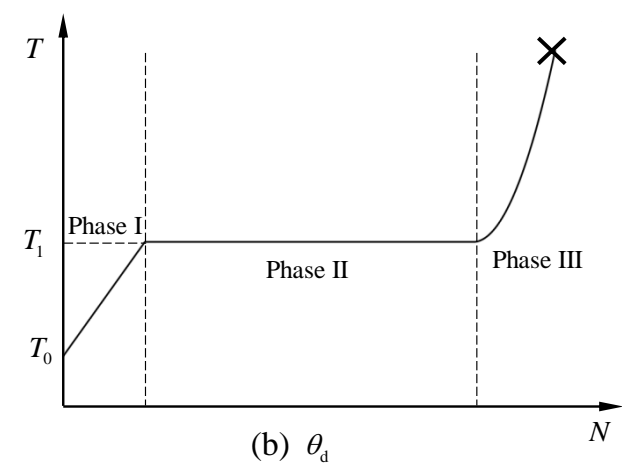

(b) $\theta_{\mathrm{d}}$

Figure 5. Schematic of three thermodynamic phases in a fatigue process: (a) temperature variation induced by both the thermoelastic coupling effect and the energy dissipation effect, and (b) temperature variation induced by the energy dissipation effect. 
The thermoelastic coupling source can be further defined as follows:

$$
s_{\text {the }}=-\alpha_{T} T_{0} E \frac{\partial(\operatorname{Trace}(\epsilon))}{\partial t}
$$

where $\epsilon$ denotes the local strain, $T_{0}$ is the ambient equilibrium temperature, $\alpha_{T}$ is the linear thermal expansion coefficient, and $E$ is Young's modulus. For sinusoidal cyclic loading, the temperature variation $\theta_{\text {the }}$ can be expressed as follows:

$$
\theta_{\text {the }}(t)=-k \frac{\alpha_{T} T_{0} E \Delta \epsilon}{\rho C} \sin \left(2 \pi f_{L}+\phi\right)
$$

where $k$ is a proportionality coefficient that can be measured by experimentation, $\Delta \epsilon$ is the strain measured by DIC system, $t$ is the loading time, and $f_{L}$ is the loading frequency. By observing Equation (7), we can obtain that $\theta_{\text {the }}$ has no contribution to the average temperature, while the temperature shift is mainly caused by $\theta_{\mathrm{d}}$. Thus, by subtracting $\theta_{\text {the }}(t)$ from the recorded temperature signal $R(t)$, we can have $\theta_{\mathrm{d}}(t)$ :

$$
\theta_{\mathrm{d}}(t)=R(t)-\theta_{\text {the }}(t)
$$

It should be noted that the specimens used in the present fatigue tests are dog-bone shaped, which can be assumed as a one-dimensional thermal diffusion problem [33]:

$$
\rho C\left(\frac{\partial \bar{\theta}_{d}(x, t)}{\partial t}+\frac{\bar{\theta}_{d}(x, t)}{\tau_{2 D}}\right)-\alpha_{T}\left(\frac{\partial^{2} \bar{\theta}_{d}(x, t)}{\partial x^{2}}\right)=\bar{d}_{1}(t)
$$

where $x$ is the distance along the loading direction, $\bar{\theta}_{d}$ is the average temperature rise induced by energy dissipation over a cross section at distance $x, \tau_{2 D}$ is the time constant characterizing heat losses through the surfaces and both ends of the specimen, and $\bar{d}_{1}$ is the average intrinsic dissipation over a cross section. It is easy to know that, in Phase II, the temperature field remains stable, so $\frac{\partial \bar{\theta}_{d}(x, t)}{\partial t}=0$. Therefore, Equation (9) becomes the following:

$$
\rho C\left(\frac{\bar{\theta}_{d}(x, t)}{\tau_{2 D}}\right)-\alpha_{T}\left(\frac{\partial^{2} \bar{\theta}_{d}(x, t)}{\partial x^{2}}\right)=\bar{d}_{1}(t)
$$

By solving differential function, the general solution of Equation (10) is obtained:

$$
\bar{\theta}_{d}(x, t)=C_{1}(t) e^{r x}+C_{2}(t) e^{-r x}+C_{3}(t)
$$

where Equation (11) characterizes the distribution of the temperature increase, in which $C_{1}$ and $C_{2}$ are coefficients of the temperature boundary condition, $C_{3}$ is the coefficient of intrinsic dissipation, and $r$ is the coefficient of heat losses through the specimen surfaces. $C_{3}$ and $r$ are defined as follows:

$$
\begin{gathered}
C_{3}(t)=\frac{\tau_{2 D} \bar{d}_{1}(t)}{\rho C} \\
r=\sqrt{\frac{\rho C}{\alpha_{T} \tau_{2 D}}}
\end{gathered}
$$

Based on temperature increase measured by Equation (8) in Phase II, the value of $C_{3}$ and $r$ are obtained by fitting the data of Equation (11). Therefore, the intrinsic dissipation rate $d_{1}(t)$ can be calculated by the following:

$$
\bar{d}_{1}(t)=\alpha_{T} r^{2} C_{3}(t)
$$




\section{Results and Discussion}

As illustrated in Figure 6, the results of the quasi-static tensile tests show that the Ultimate Tensile Stresses (UTS) of GC and LPBF AlSi10Mg alloys are 328.3 MPa and 435.2 $\mathrm{MPa}$, respectively. Therefore, the strength of LPBF AlSi10Mg alloy is significantly larger than that of casting, which is in accordance with the existing literature [3,4]. The values of Young's modulus for GC and LPBF AlSi10Mg alloys are measured as $69.3 \mathrm{GPa}$ and $71.2 \mathrm{GPa}$, respectively. The elongations of the GC and LPBF AlSi10Mg alloys are tested as $13.26 \%$ and $8.45 \%$, respectively. It is because the temperature changes rapidly during LPBF processing, which results in smaller grains and more grain boundaries. Thus, it is difficult for dislocation to cross grain boundaries, which leads to a higher strength. In the meantime, the defects of pores and incompleted melting lead to lower elongation.

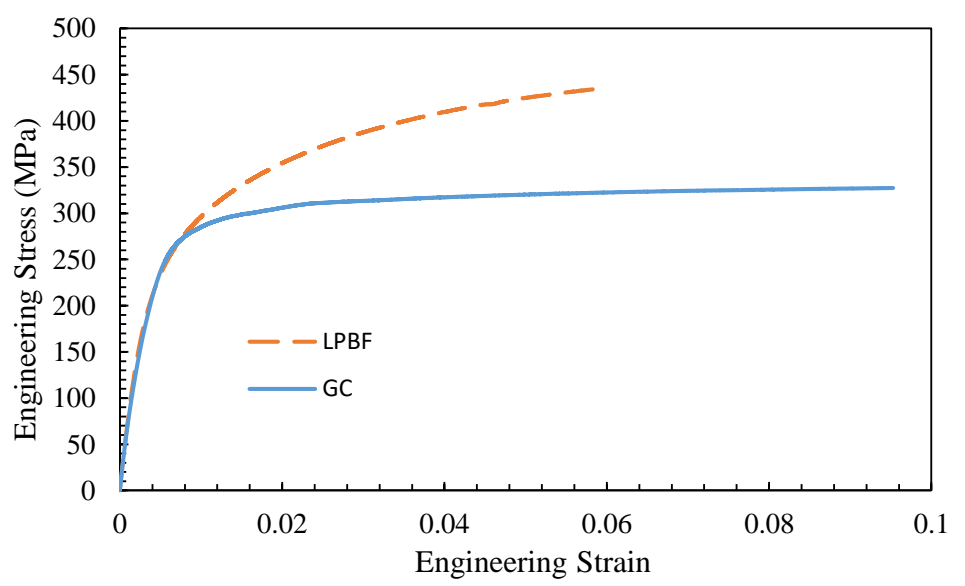

Figure 6. Experimental results of the uniaxial tensile tests for GC and LPBF AlSi10Mg.

A typical image captured by IR camera is shown in Figure 7. As can be seen in the figure, the surface temperature of the testing specimen has almost no difference from the reference specimen at the beginning of the fatigue test. After 3000 loading cycles, the surface temperature of the testing specimen is obviously higher than the surroundings and the reference specimen, which shows the phenomenon of self-heating. The right zone of the red outline records the surface temperature of the gauge section of the specimen under fatigue load and the left zone of the red outline records the surface temperature of the reference specimen, presented side by side. The difference is the temperature values in those two zones is identified as the relative temperature increase $R(t)$.
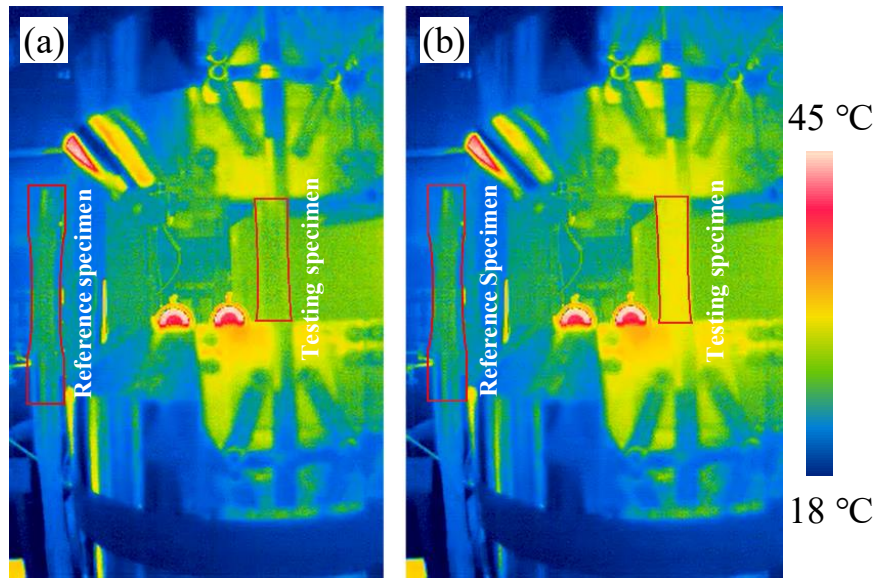

Figure 7. A typical image of the temperature field of the testing specimen and reference specimen: (a) temperature field at the beginning of the test; (b) temperature field after 3000 cycles. 
Figure 8 presents an example of the signal processing result for the LPBF AlSi10Mg alloy under the stress level of $75 \%$ UTS to separate the thermoelastic coupling effect $\theta_{\text {the }}(t)$ and energy dissipation effect $\theta_{\mathrm{d}}(t)$. We can observe that the original recording data contains both $\theta_{\text {the }}(t)$ and $\theta_{\mathrm{d}}(t)$ Figure 8a. According to Equations (6)-(8), the temperature variation induced by the thermoelastic coupling effect is a sinusoidal function and that the amplitude is proportionate to the maximum local strain. By setting the same sampling frequency, the signal of $\theta_{\text {the }}(t)$ can be obtained. It can be seen that the temperature variation in Figure $8 \mathrm{~b}$ fluctuates between $0.6{ }^{\circ} \mathrm{C}$ to $-0.6{ }^{\circ} \mathrm{C}$. By subtracting the signal of Figure $8 \mathrm{~b}$ from the signal of Figure $8 \mathrm{a}$, we can observe that the temperature shift is caused by the energy dissipation effect $\theta_{\mathrm{d}}(t)$. As shown in Figure $8 \mathrm{c}$, the value of $\theta_{\mathrm{d}}(t)$ keeps increasing before $150 \mathrm{~s}$, while it gradually become stable after $150 \mathrm{~s}$. Thus, the average temperature increase in the last $50 \mathrm{~s} \overline{\bar{\theta}}_{\mathrm{d}}$ can be identified as a stable temperature increase caused by energy dissipation.
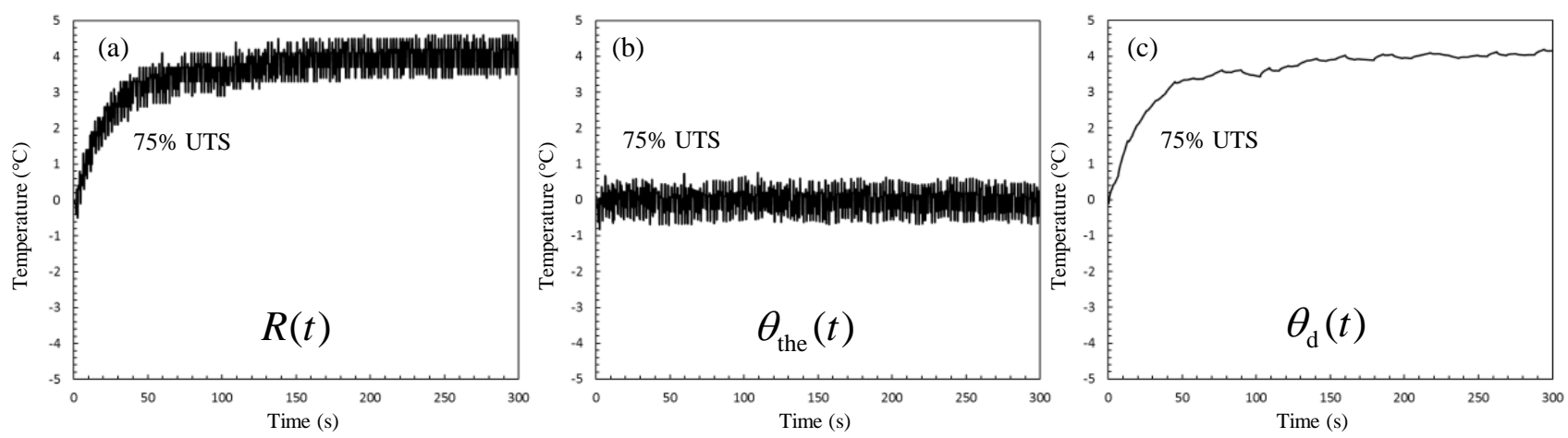

Figure 8. Separation of the thermoelastic coupling effect and energy dissipation effect for LPBF AlSi10Mg alloy under the stress level of $75 \%$ Ultimate Tension Stress (UTS). (a) Relative temperature increase acquired by the IR camera, (b) temperature variation caused by the thermoelastic coupling effect, and (c) temperature shift caused by the energy dissipation effect.

After processing all of the data of the temperature signal for the GC and LPBF specimens under different applied stress magnitudes, the stable temperature increase subjected to each loading magnitude can be obtained. Figure 9 shows the plots of the stable temperature increase as a function of the applied stress magnitudes for both GC and LPBF AlSi10Mg alloys. According to previous research work [9,14,36-38], the turning point of the relationship between stable temperature rising and applied stress magnitudes can be considered the fatigue limit. Recent research [39] shows that this turning point is where a change in the strain mechanisms occurs. The permanent dislocation movement determines an unrecoverable deformation status of the material. An optimized graphic method [17] is employed here to capture this turning point. The relationship curve is characterized by two straight lines, and the intersection of the two lines can be considered the turning point. The cyclic loading stress related to this point can be taken as the fatigue limit (failure cycle number larger than $10^{6}$ ). As can be seen in Figure 9, the fatigue limits of the GC and LPBF AlSi10Mg alloys are determined as 55.8\% UTS (183.2 MPa) and 33.9\% UTS (147.5 MPa), respectively. The predicted results are used for the separation of energy dissipation related to irreversible degradation and internal friction. 

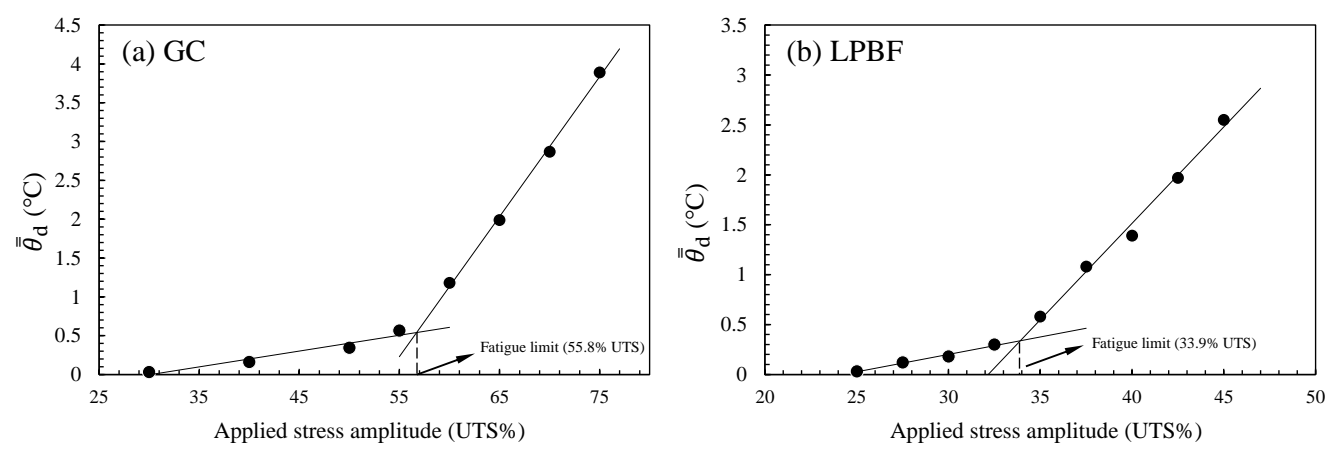

Figure 9. Fatigue limit prediction based on the relationship of the average temperature shift caused by energy dissipation: (a) GC specimen; (b) LPBF specimen.

As mentioned in Section 3, a one-dimensional double exponential regression model (see Equation (11)) can be used to calculate the energy dissipation rate per unit volume. Figure 10 shows an example of the GC AlSi10Mg alloy under the applied stress amplitude of 75\% UTS. The plot gives the data of a stable temperature increase distributed along the $x$ axis (loading direction). $x=0$ is the position of the center of gauge section. The temperature data are obtained by processing the infrared thermal image, which contains 51 data points in total, corresponding to the gauge section from $-10 \mathrm{~mm}$ to $8 \mathrm{~mm}$. It can be observed that the location of the highest temperature increase was not strictly in the middle of the specimen, which is mainly caused by the inconsistency of the temperature boundary conditions at both ends of the test specimen in the fatigue experiment. This does not affect the precision of the calculation of the regression model. The R-square of this regression case is 0.9824 , and the sum of squares due to error is 0.005185 , which indicates that the one-dimensional double exponential regression model can describe the axial distribution of temperature well. In the meantime, all of the parameters of the regression model can be determined and the energy dissipation rate can be calculated by Equation (14). The average energy dissipation rate $\overline{\bar{d}}_{1}$ is computed as the mean value of the last $50 \mathrm{~s}$.

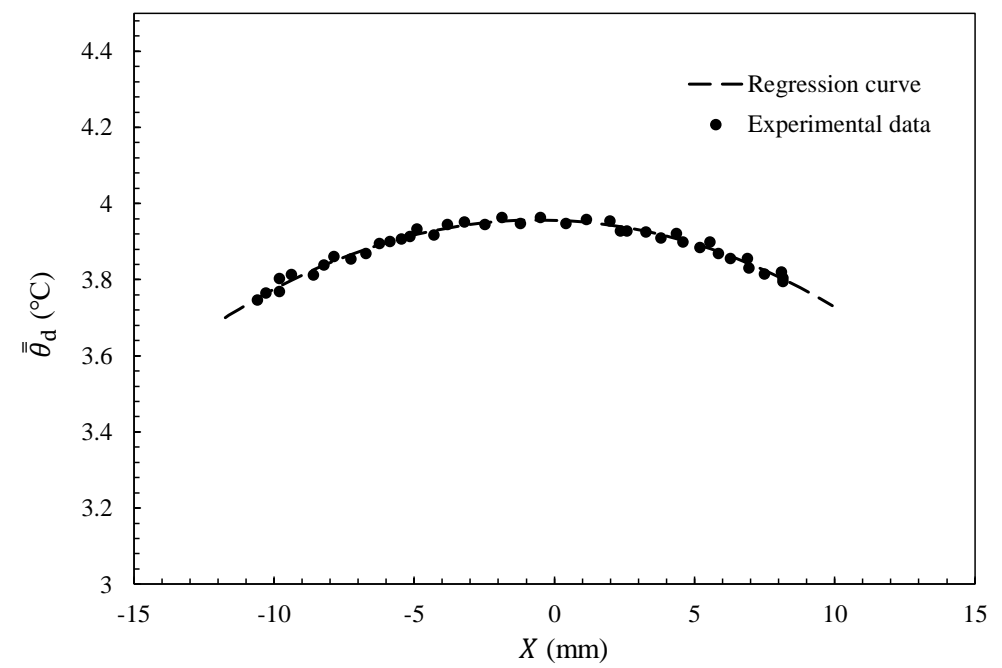

Figure 10. One-dimensional distribution of the average temperature shift and the double exponential regression curve.

Based on a double exponential regression model, the relationships between $\overline{\bar{d}}_{1}$ and the applied stress amplitude of the GC and LPBF AlSi10Mg alloys are shown in Figure 11. The plot shows that the value of $\overline{\bar{d}}_{1}$ increases with the maximum loading amplitude for both GC and LPBF AlSi10Mg alloys. We can observe that the average energy dissipation rate of 
LPBF AlSi10Mg is larger than that of the GC AlSi10Mg alloy under the same applied stress amplitude, which indicates that the data of energy dissipation can be used to distinguish the fatigue damage evolution behavior of the GC and LPBF AlSi10Mg alloys. As mentioned in the Section 1, the energy dissipation can be induced by internal friction and irreversible changes, in which only the latter is related to fatigue damage. In order to figure out the energy dissipation rate only, caused by irreversible changes (damage accumulation), the predicted fatigue limit obtained in Figure 9 is used to separate the relationship curve into two parts. The energy dissipation of specimens cyclically loaded below the predicted fatigue limit is assumed to be only caused by internal friction, while the energy dissipation corresponding to loading amplitude beyond the predicted fatigue limit is caused by both internal friction and damage accumulation. According to previous research $[17,18]$, the internal friction is mainly derived from the displacement of microstructure motion and the intensity of the stress. Therefore, the authors used a polynomial function to characterize energy dissipation of internal friction effect. The data from the energy dissipation rate below the fatigue limit were used to fit the polynomial function, and the data from the energy dissipation rate beyond the fatigue limit were accordingly estimated. Under this assumption, the total energy dissipation rate was separated into two parts, as shown in Figure 11. It can be seen that the internal friction effect dominated under low applied stress amplitude, while the energy dissipation related to fatigue damage becomes significantly larger than internal friction under a high applied stress amplitude, which is similar to the results in previous research $[31,33,40]$. By comparing Figure 11a,b, we can also observe that, under the same high applied stress amplitude, the energy dissipation related to fatigue damage for LPBF specimens is obviously higher than that of GC specimens, which indicates that the fatigue property of LPBF specimen is worse than that of the GC specimen.
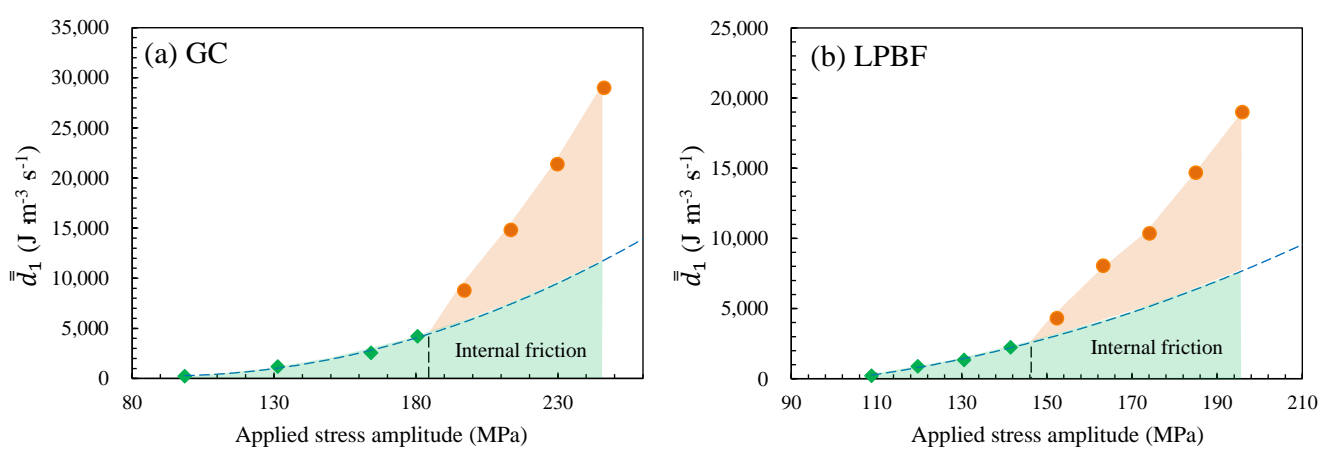

Figure 11. Separation of the energy dissipation rate induced by internal friction and irreversible degradation: (a) GC AlSi10Mg alloy; (b) LPBF AlSi10Mg alloy.

In order to quantificationally study the fatigue property based on the data of energy dissipation, the concept of fatigue damage entropy is introduced here. The hypothesis of damage entropy thinks that the fatigue damage evolution is an entropy-increasing process accompanied by energy dissipation. Therefore, the fatigue damage entropy $\Gamma_{\text {damage }}$ can be computed by the following:

$$
\Gamma_{\text {damage }}=\frac{\overline{\bar{d}}_{1 \text { damage }} N_{f}}{f_{L} T_{1}}
$$

where $\overline{\bar{d}}_{1 \text { damage }}$ is the average energy dissipation rate related to fatigue damage; $N_{f}$ is the failure cycle number; and $T_{1}$ is the stable temperature rising, which is shown in Figure 5. Previous research [25-27] show that the value of fatigue entropy was proven to be independent of applied stress amplitude, so the fatigue life can be evaluated by transforming Equation (15):

$$
N_{f}=\frac{f_{L} T_{1} \Gamma_{\text {damage }}}{\overline{\bar{d}}_{1 \text { damage }}}
$$


By carrying out fatigue test for one specimen until final fracture in order to calculate the value of fatigue damage entropy, the S-N curves of both GC and LPBF AlSi10Mg alloys can be predicted as shown in Figure 12. The traditional experimental S-N curve of GC and LPBF AlSi10Mg alloys from Reference [4] are also given for comparison. It can be seen that the predicted S-N curve matches well with the experimental one for LPBF AlSi10Mg alloys, while the prediction of GC AlSi10Mg alloy is conservative compared with traditional experimentation. On the whole, the prediction can make a clear distinction of the fatigue property of GC and LPBF AlSi10Mg alloys in a short time (less than $48 \mathrm{~h}$ ), and the precision is also acceptable for quick evaluation.

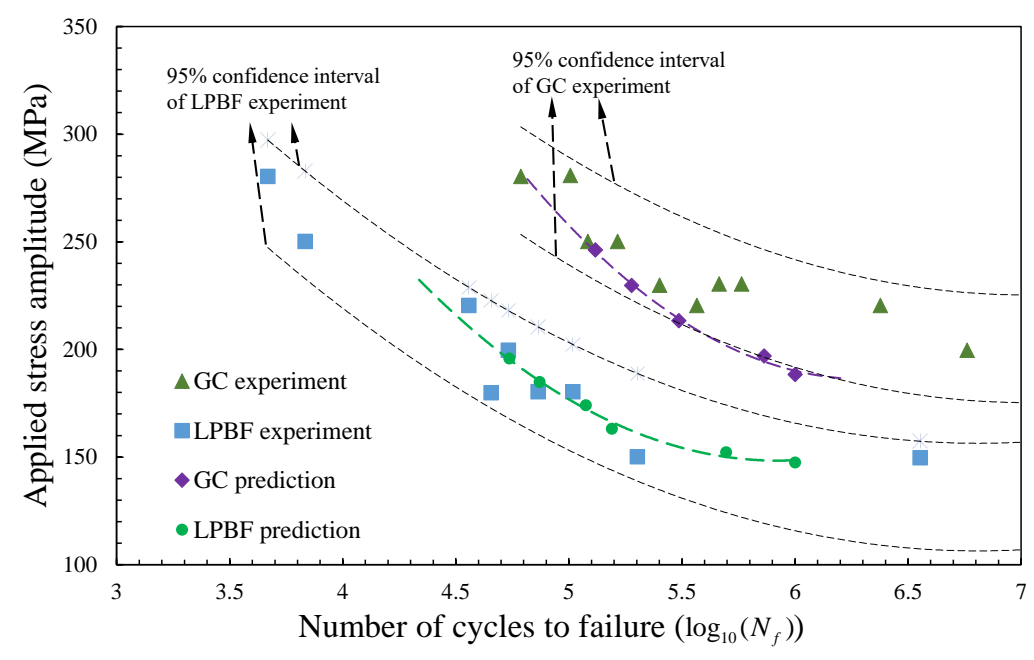

Figure 12. Comparison of predicted S-N curves and traditional fatigue testing results adapted from [4] for both Gravity Casting (GC) and Laser-based Powder Bed Fusion (LPBF) AlSi10Mg alloys.

\section{Conclusions}

In this paper, the fatigue energy dissipation of GC and LPBF AlSi10Mg alloys subjected to fatigue loading are investigated and the research results can be used to evaluate the fatigue property efficiently. The main remarks are concluded below:

1. The signal of surface temperature variation corresponding to the thermoelastic coupling effect and the energy dissipation effect is decoupled. The temperature increase induced by energy dissipation effect is used to the predict fatigue limit of GC and LPBF AlSi10Mg alloys by two linear fitting methods. The fatigue limits of GC and LPBF AlSi10Mg alloys are determined as 55.8\% UTS and 33.9\% UTS, respectively.

2. The energy dissipation rates of GC and LPBF AlSi10Mg alloys are obtained by solving a one-dimensional thermal diffusion problem. The amount of energy dissipation caused by internal friction and fatigue damage is separated using a polynomial function. The energy dissipation related to fatigue damage for LPBF specimens is observed to be higher than that of GC specimens.

3. The concept of fatigue damage entropy is employed to predict the fatigue life of both GC and LPBF AlSi10Mg alloys based on the obtained energy dissipation data. The prediction has a good agreement with the experimental results. The whole prediction process takes less than $48 \mathrm{~h}$, which is significantly less than traditional fatigue testing.

Nevertheless, the direct correlation between the energy dissipation and microstructure change for GC and LPBF AlSi10Mg alloys is still unclear, which needs further studies with in-suit thermodynamic experiments. In addition, the model in this paper also has the potential to rapidly assess the quality of 3D-printed structures. 
Author Contributions: Conceptualization, C.Y., J.H., K.Z. (Ke Zhu) and Y.L.; investigation, C.Y., W.L. and K.Z. (Ke Zhu); writing-original draft preparation, C.Y., Y.C. and J.H.; writing-review and editing, K.Z. (Ke Zhu), Y.L., K.Z. (Keming Zhang) and W.L. All authors read and agreed to the published version of the manuscript.

Funding: This paper was sponsored by Chinese Special Program of Civil Aircraft, the National Natural Science Foundation of China (grant number 11802316 and grant number 12002211) and the Shanghai Sailing Program (No. 20YF1432700).

Institutional Review Board Statement: Not applicable.

Informed Consent Statement: Not applicable.

Data Availability Statement: Data sharing is not applicable to this article.

Conflicts of Interest: The authors declare no conflict of interest.

\section{References}

1. Bhuvanesh Kumar, M.; Sathiya, P. Methods and materials for additive manufacturing: A critical review on advancements and challenges. Thin-Walled Struct. 2021, 159, 107228. [CrossRef]

2. Rasiya, G.; Shukla, A.; Saran, K. Additive Manufacturing-A Review. Mater. Today Proc. 2021. [CrossRef]

3. Roth, C.C.; Tancogne-Dejean, T.; Mohr, D. Plasticity and fracture of cast and SLM AlSi10Mg: High-throughput testing and modeling. Addit. Manuf. 2021, 43, 101998. [CrossRef]

4. Yan, Q.; Song, B.; Shi, Y. Comparative study of performance comparison of AlSi10Mg alloy prepared by selective laser melting and casting. J. Mater. Sci. Technol. 2020,41, 199-208. [CrossRef]

5. Shakil, S.; Hadadzadeh, A.; Shalchi Amirkhiz, B.; Pirgazi, H.; Mohammadi, M.; Haghshenas, M. Additive manufactured versus cast AlSi10Mg alloy: Microstructure and micromechanics. Results Mater. 2021, 10, 100178. [CrossRef]

6. Luong, M.P. Infrared thermographic scanning of fatigue in metals. Nucl. Eng. Des. 1995, 158, 363-376. [CrossRef]

7. La Rosa, G.; Lo Savio, F.; Giudice, F.; Clienti, C.; Marino Cugno Garrano, A. Energetic analysis of fatigue hysteresis by thermographic and digital image correlation methodologies. Fatigue Fract. Eng. Mater. Struct. 2020, 43, 2597-2607. [CrossRef]

8. La Rosa, G.; Risitano, A. Thermographic methodology for rapid determination of the fatigue limit of materials and mechanical components. Int. J. Fatigue 2000, 22, 65-73. [CrossRef]

9. Luong, M.P. Fatigue limit evaluation of metals using an infrared thermographic technique. Mech. Mater. 1998, 28, 155-163. [CrossRef]

10. Fargione, G.; Geraci, A.; La Rosa, G.; Risitano, A. Rapid determination of the fatigue curve by the thermographic method. Int. J. Fatigue 2002, 24, 11-19. [CrossRef]

11. Morabito, A.; Chrysochoos, A.; Dattoma, V.; Galietti, U. Analysis of heat sources accompanying the fatigue of 2024 T3 aluminium alloys. Int. J. Fatigue 2007, 29, 977-984. [CrossRef]

12. Meneghetti, G.; Ricotta, M. Evaluating the heat energy dissipated in a small volume surrounding the tip of a fatigue crack. Int. J. Fatigue 2016, 92, 605-615. [CrossRef]

13. Ancona, F.; De Finis, R.; Palumbo, D.; Galietti, U. Crack growth monitoring in stainless steels by means of TSA technique. Procedia Eng. 2015, 109, 89-96. [CrossRef]

14. Munier, R.; Doudard, C.; Calloch, S.; Weber, B. Determination of high cycle fatigue properties of a wide range of steel sheet grades from self-heating measurements. Int. J. Fatigue 2014, 63, 46-61. [CrossRef]

15. De Finis, R.; Palumbo, D.; Ancona, F.; Galietti, U. Fatigue limit evaluation of various martensitic stainless steels with new robust thermographic data analysis. Int. J. Fatigue 2015, 74, 88-96. [CrossRef]

16. Meneghetti, G. Analysis of the fatigue strength of a stainless steel based on the energy dissipation. Int. J. Fatigue 2007, 29, 81-94. [CrossRef]

17. Huang, J.; Pastor, M.L.; Garnier, C.; Gong, X. Rapid evaluation of fatigue limit on thermographic data analysis. Int. J. Fatigue 2017, 104, 293-301. [CrossRef]

18. Huang, J.; Pastor, M.L.; Garnier, C.; Gong, X.J. A new model for fatigue life prediction based on infrared thermography and degradation process for CFRP composite laminates. Int. J. Fatigue 2019, 120, 87-95. [CrossRef]

19. Curà, F.; Curti, G.; Sesana, R. A new iteration method for the thermographic determination of fatigue limit in steels. Int. J. Fatigue 2005, 27, 453-459. [CrossRef]

20. Amiri, M.; Khonsari, M.M. Life prediction of metals undergoing fatigue load based on temperature evolution. Mater. Sci. Eng. A 2010, 527, 1555-1559. [CrossRef]

21. De Finis, R.; Palumbo, D.; Ancona, F.; Galietti, U. Fatigue behaviour of stainless steels: A multi-parametric approach. In Residual Stress, Thermomechanics \& Infrared Imaging, Hybrid Techniques and Inverse Problems; Springer: Berlin/Heidelberg, Germany, 2017; Volume 9, pp. 1-8.

22. Pitarresi, G. Lock-in signal post-processing techniques in infra-red thermography for materials structural evaluation. Exp. Mech. 2015, 55, 667-680. [CrossRef] 
23. Naderi, M.; Amiri, M.; Khonsari, M.M. On the thermodynamic entropy of fatigue fracture. Proc. Math. Phys. Eng. Sci. 2010, 466, 423-438. [CrossRef]

24. Naderi, M.; Khonsari, M.M. Thermodynamic analysis of fatigue failure in a composite laminate. Mech. Mater. 2012, 46, 113-122. [CrossRef]

25. Liakat, M.; Khonsari, M.M. Rapid estimation of fatigue entropy and toughness in metals. Mater. Des. 2014, 62, 149-157. [CrossRef]

26. Liakat, M.; Khonsari, M.M. On the anelasticity and fatigue fracture entropy in high-cycle metal fatigue. Mater. Des. 2015, 82, 18-27. [CrossRef]

27. Mehdizadeh, M.; Khonsari, M.M. On the application of fracture fatigue entropy to variable frequency and loading amplitude. Theor. Appl. Fract. Mech. 2018, 98, 30-37. [CrossRef]

28. Mahmoudi, A.; Mohammadi, B. On the evaluation of damage-entropy model in cross-ply laminated composites. Eng. Fract. Mech. 2019, 219, 106626. [CrossRef]

29. Mohammadi, B.; Mahmoudi, A. Developing a new model to predict the fatigue life of cross-ply laminates using coupled CDM-entropy generation approach. Theor. Appl. Fract. Mech. 2018, 95, 18-27. [CrossRef]

30. Calleja-Ochoa, A.; Gonzalez-Barrio, H.; Lacalle, N.L.d.; Martínez, S.; Albizuri, J.; Lamikiz, A. A New Approach in the Design of Microstructured Ultralight Components to Achieve Maximum Functional Performance. Materials 2021, 14, 1588. [CrossRef] [PubMed]

31. Guo, Q.; Guo, X. Research on high-cycle fatigue behavior of FV520B stainless steel based on intrinsic dissipation. Mater. Des. 2016, 90, 248-255. [CrossRef]

32. Maquin, F.; Pierron, F. Heat dissipation measurements in low stress cyclic loading of metallic materials: From internal friction to micro-plasticity. Mech. Mater. 2009, 41, 928-942. [CrossRef]

33. Guo, Q.; Guo, X.; Fan, J.; Syed, R.; Wu, C. An energy method for rapid evaluation of high-cycle fatigue parameters based on intrinsic dissipation. Int. J. Fatigue 2015, 80, 136-144. [CrossRef]

34. Boulanger, T. Calorimetric analysis of dissipative and thermoelastic effects associated with the fatigue behavior of steels. Int. J. Fatigue 2004, 26, 221-229. [CrossRef]

35. Chrysochoos, A.; Louche, H. An infrared image processing to analyse the calorific effects accompanying strain localisation. Int. J. Eng. Sci. 2000, 38, 1759-1788. [CrossRef]

36. Montesano, J.; Fawaz, Z.; Bougherara, H. Use of infrared thermography to investigate the fatigue behavior of a carbon fiber reinforced polymer composite. Compos. Struct. 2013, 97, 76-83. [CrossRef]

37. Kordatos, E.; Dassios, K.; Aggelis, D.; Matikas, T. Rapid evaluation of the fatigue limit in composites using infrared lock-in thermography and acoustic emission. Mech. Res. Commun. 2013, 54, 14-20. [CrossRef]

38. Bagheri, Z.S.; El Sawi, I.; Bougherara, H.; Zdero, R. Biomechanical fatigue analysis of an advanced new carbon fiber/flax/epoxy plate for bone fracture repair using conventional fatigue tests and thermography. J. Mech. Behav. Biomed. Mater. 2014, 35, 27-38. [CrossRef] [PubMed]

39. Nourian-Avval, A.; Khonsari, M. Rapid prediction of fatigue life based on thermodynamic entropy generation. Int. J. Fatigue 2021, 145, 106105. [CrossRef]

40. Mehdizadeh, M.; Khonsari, M.M. On the role of internal friction in low-and high-cycle fatigue. Int. J. Fatigue 2018, 114, 159-166. [CrossRef] 